\title{
Load Reduction Potential of Variable Speed Control Approaches for Fixed Pitch Tidal Current Turbines
}

\author{
Matthias Arnold $^{\# 1}$, Frank Biskup ${ }^{* 2}$, Po Wen Cheng ${ }^{\# 3}$ \\ ${ }^{\#}$ Stuttgart Wind Energy (SWE), University Stuttgart \\ Allmandring 5b, 70569 Stuttgart, Germany \\ ${ }^{1}$ Arnold@ifb.uni-stuttgart.de \\ ${ }^{3}$ Cheng@ifb.uni-stuttgart.de \\ "Voith Hydro Ocean Current Technologies GmbH \& Co. KG \\ Alexanderstr. 11, 89522 Heidenheim, Germany \\ ${ }^{2}$ Frank.Biskup@voith.com
}

\begin{abstract}
The present paper compares control strategies for fixed pitch Tidal Current Turbines and introduces the underspeed control technique. The state of the art approach of overspeed control for fixed pitch turbine increases the tip speed ratio to limit the power output of the turbine. This leads to a high fatigue loading as the number of load cycles increases and requires a specific rotor design not to increase the thrust coefficient as well.

The underspeed controller on the other side reduces the tip speed ratio resulting in a reduced thrust coefficient and a reduced number of load cycles. However, this point of operation is unstable based on the characteristic curves of the rotor and needs to be stabilized by the controller. The basic structure of the controller as well as the calculated stability limits will be shown in the paper.

Further, the performance of the underspeed controller regarding loads is compared to state of the art fixed and variable pitch controllers. The paper concludes with an analysis of the impacts on the turbine design and further potential and issues arising with the underspeed control approach.
\end{abstract}

Keywords Tidal Energy, Fixed Pitch Tidal Current Turbine, Underspeed Controller, Stall, Variable Speed, Turbine Design, Hydrodynamics, Dynamic Loads

\section{INTRODUCTION}

To limit the loads on a tidal current turbine it is common practice to define a rated power $P_{\text {rated }}$ above which the turbine is no more producing optimal power. This limitation of turbine power is achieved by shifting the operating point to one with reduced efficiency with the controller. The point of operation is hereby defined by the required power coefficient $c_{P, \text { req. }}\left(v_{1}\right)$ depending on the current velocity $v_{1}$,

$$
c_{P, \text { req. }}\left(v_{1}\right)=\frac{P_{\text {Rated }}}{\frac{\rho}{2} \pi R_{\text {Rotor }}^{2} \cdot v_{1}^{3}} .
$$

Comparing this value to the characteristics of the rotor $c_{P}\left(\lambda_{T S R}, \beta\right)$, a range of tip speed ratios $\lambda_{T S R}$ and pitch angles $\beta$ can be found being possible points of operation, cf. Fig. 1 .

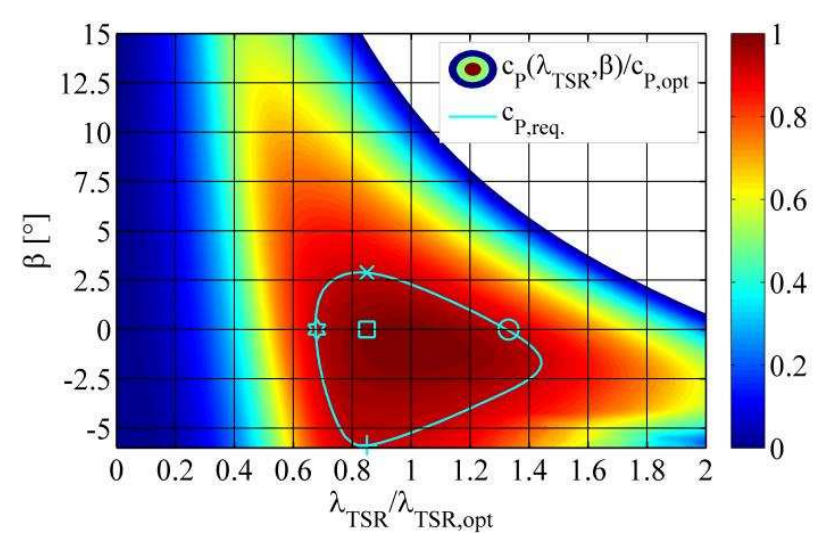

Fig. 1: Power coefficient $c_{P}\left(\lambda_{T S R}, \beta\right)$ with exemplary point of operation $c_{P, \text { req. }}$

\section{A. State of the Art}

As sketched in Fig. 1 there are 3 typical ways to achieve the required $c_{P, r e q}$. on tidal current turbines - 'Overspeed' $(0)$, 'Active Stall' (+) and 'Pitch' (x). Each of these options has its specific advantages and disadvantages.

Pitch, and in former times active stall, are typical concepts in wind energy, [8]. Here the blade is rotated resp. pitched to influence the angle of attack $\alpha_{A O A}$ on each radial position, resulting in a limitation of fatigue and ultimate loads, [13]. This approach is also versatile regarding load reduction with e.g. active tower damping, [9], individual pitch [6] or predicted control, [19]. However, it relies on the functionality of the pitch actuator, a component prone to failure, [22].

On the other hand, the category of fixed pitch turbines doesn't use a pitch actuator, but heads for a maximum of reliability accepting higher loads, [18], [20], [21]. The turbine has to be designed sturdier therefore increasing the bill of material but limiting the operational costs. However, following the approach of a fixed pitch turbine the power output still needs to be limited to rated power. The state of the art for this concept is to increase the tip speed ratio with the overspeed controller by reducing the generator torque $Q$ above 
rated speed $\Omega_{\text {rated }}$, [1], [23], and consequently reducing the power coefficient $c_{P}$. However, this also increases the thrust coefficient $c_{T h}$ and the number of load cycles. Specific designs of the rotor blades can limit the thrust coefficient $c_{T h}$ or even slightly reduce it, [4], nevertheless there is a high fatigue loading the system has to be designed for.

\section{B. Idea of the Underspeed Controller}

Considering the advantage of the fixed pitch concept avoiding a pitch actuator system and the advantage of a pitch system with reduced loads, a combination of those is desired. Analysing the power coefficient $c_{P}$ for a fixed pitch turbine leaves 2 possible points of operation for rated power output, one above the optimal tip speed ratio, $\lambda_{\text {overspeed }}>\lambda_{\text {TSR,opt }}$, and one below, $\lambda_{\text {underspeed }}<\lambda_{\text {TSR,opt }}$. The point above rated is used for the known method of overspeed control as described above.

Operation with $\lambda_{\text {underspeed }}$, the underspeed control (cf. Fig. 1, marker: $\star$ ), features several desired advantages: rated power output, low thrust coefficient, low number of load cycles due to low rotational speed, etc. However, this approach is incompatible with the known method of applying a generator torque $Q$ as a function of the rotor speed $\Omega$, $Q=f(\Omega)$, but requires a torque dependent rotor speed, $\Omega=f(Q)$, as shown in Fig. 2. As it is required to reduce the rotor speed by increasing the electrical power to reduce the hydrodynamic power of the turbine, there is also no inherent stability in the system as known from overspeed. This makes the controller design more complex as it needs to bring this unstable system into stability.

One could claim now, that forcing the rotor into stall by reducing tip speed ratio is the known method of a stall regulated turbine, 'Danish concept' (cf. Fig. 1, marker: $\square$ ), [10], however there is a distinct difference. A turbine with the Danish concept uses a fixed speed and is therefore not able to maintain the rated power output over a range of current speeds, but the power output varies depending on the current speed. The underspeed controller on the other hand reacts with a variable speed to any change in current speed.

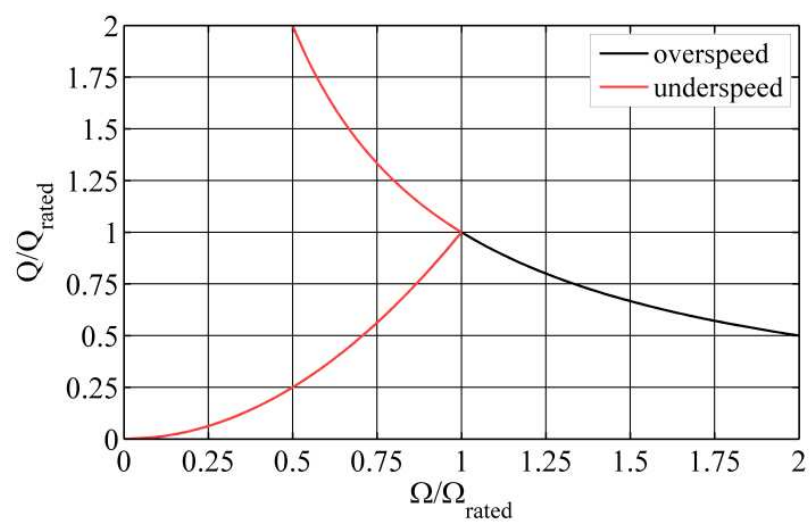

Fig. 2: Optimal generator torque for fixed pitch turbines

\section{Previous Work on the Underspeed Concept}

The underspeed controller is not a completely new idea but has been mentioned previously in literature e.g. as 'variable speed stall regulated controller'. [12] describes the method of controlling a turbine with the underspeed technique with reference to [17], however focused on direct drive wind turbines. As a wind turbine has low rotational speed and high inertia [12] claims exceptional torque values difficult to achieve with the generator not feasible for a full scale application. However, as the rotational inertias are several orders of magnitude smaller on tidal current turbines, the observed peak in required torque during a change in current speed and subsequent change in rotational speed will be much smaller. Also these investigations focused on the annual energy production, but not on the changes in loads.

For tidal current turbines the concept has been mentioned by [11]. The idea is explained and the characteristic curves are shown, however [11] gives no details on the setup, stability, properties nor dynamic responses.

\section{Investigated Turbine}

The turbine investigated here is a theoretic turbine with parameters based on the Voith HyTide ${ }^{\circledR} 1000-13$ turbine concept. While the turbine parameters shown in Table I are abstracted from the real turbine parameters including enclosed water, etc. for simplification, the integral properties of the rotor hydrodynamics are directly used with the power coefficient $c_{P \text { HyTide }}$, cf. Fig. 1, and thrust coefficient $c_{\text {Th HyTide }}$. Despite the fact this rotor is specifically designed to operate with an overspeed controller, [4], it also matches the rotor blade design targets for underspeed controlled turbines, discussed later. Being a well-rounded rotor it is therefore an equal basis for the comparison to state of the art controllers with different approaches.

TABLE I

TURBINE PROPERTIES

\begin{tabular}{|c|c|}
\hline \multicolumn{2}{|c|}{ Rotor \& Drivetrain } \\
\hline Rated Power: & Diameter: \\
\hline$P_{\text {rated }}=1 \mathrm{MW}$ & $D=13 m$ \\
\hline $\begin{array}{l}\text { Rotor hydrodynamics } \\
\begin{aligned} c_{P}\left(\lambda_{T S R}, \beta\right) & =c_{P} \text { HyTide } \\
c_{T h}\left(\lambda_{T S R}, \beta\right) & =c_{\text {Th HyTide }}\end{aligned}\end{array}$ & $\begin{array}{l}\text { Inertia (including added } \\
\text { mass) } \\
\qquad J=500 \cdot 10^{3} \mathrm{kgm}^{2}\end{array}$ \\
\hline \multicolumn{2}{|c|}{$\begin{array}{l}\text { Nacelle \& Foundation } \\
\end{array}$} \\
\hline $\begin{array}{l}\text { Nacelle Volume } \\
\qquad V_{\text {Nacelle }}=81.7 \mathrm{~m}^{3}\end{array}$ & $\begin{array}{l}\text { Tower stiffness } \\
\quad c=10 \cdot 10^{6} \mathrm{~N} / \mathrm{m}\end{array}$ \\
\hline $\begin{array}{l}\text { Nacelle drag } \\
\qquad A \cdot c_{d}=10 \mathrm{~m}^{2}\end{array}$ & $\begin{array}{c}\text { Nacelle added mass coeff. } \\
C_{a}=0.133 \\
\end{array}$ \\
\hline $\begin{array}{l}\text { Equivalent tower head mass } \\
\text { tower mass fraction, etc.) } \\
\qquad m_{\text {Heac }}\end{array}$ & $\begin{array}{l}\text { icluding enclosed water, } \\
=270 t\end{array}$ \\
\hline
\end{tabular}

\section{UNDERSPEED CONTROLLER}

\section{A. Steady Operational Points}

Fig. 3 shows the exemplary steady points of operation with increasing current speed. As can be seen the tip speed ratio is kept constant for below rated, optimal power production, and 
significantly reduced for above rated operation. This leads to a constant power and reduced thrust as desired.

However there is also a first hint to a practical issue noticeable for the underspeed control, which needs to be addressed. Right after the rated point, the rotor speed needs to be significantly reduced to maintain rated power. In transient cases this can lead to high speed and power fluctuations. The characteristic curve of the controller, cf. Fig. 2, requires therefore a modification as will be shown later.

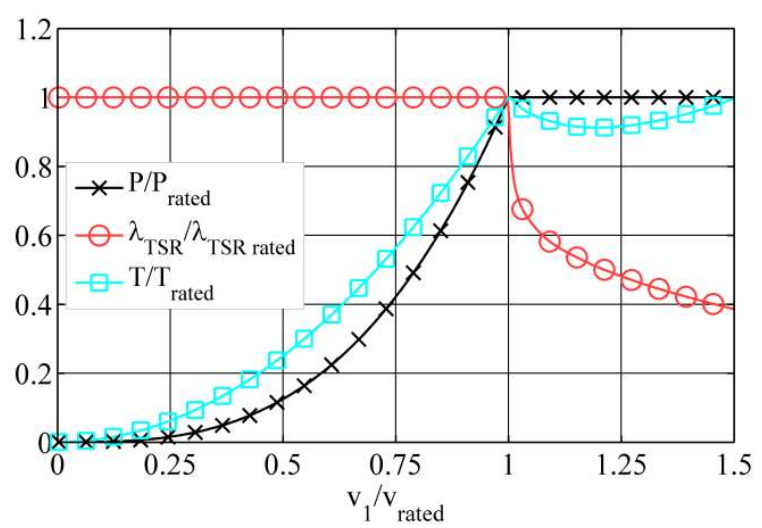

Fig. 3: Points of steady operation for an underspeed controlled turbine

\section{B. Layout of Controller}

As mentioned above the controller needs to achieve an optimal speed $\Omega_{o p t}$ as a function of the generator torque $Q_{g e n}$ during operation. The resultant setup is shown in Fig. 4. It consists of the turbine with the input of hydrodynamic torque $Q_{\text {Hydro }}$ and generator torque $Q_{g e n}$ and rotational speed $\Omega$ as output. The generator torque $Q_{g e n}$ is controlled by a PIcontroller and applied to the turbine and the transfer function $G_{Q_{g e n} \rightarrow \Omega_{r e q}}$ calculating the required speed $\Omega_{r e q}$ as input to the PI-controller. The required behaviour of $G_{Q_{g e n} \rightarrow \Omega_{\text {req }}}$ is determined below within the stability analysis.

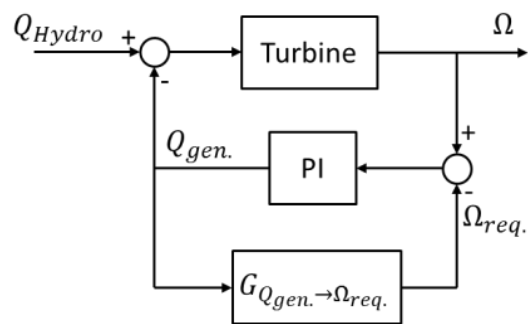

Fig. 4: Layout of controller $Q_{H y d r o} \rightarrow \Omega$

\section{Inner Closed-Loop Stability}

To analyse the controller it can be split in an inner and an outer loop. The inner loop contains the turbine and a PIcontroller as shown in Fig. 5. Its purpose is to control the turbine rotational speed $\Omega$ to a set point value $\Omega_{r e q}$. This loop needs to be stable for all possible points of operation.

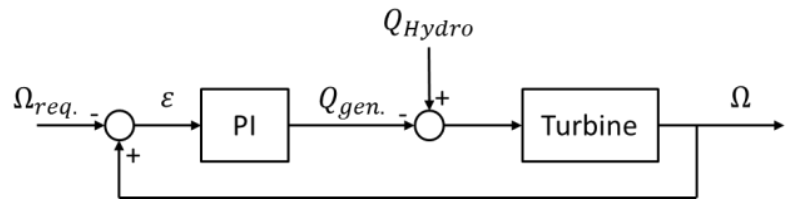

Fig. 5: Inner loop of controller $\Omega_{\text {req. }} \rightarrow \Omega$, extracted from upper half of Fig. 4

By introducing the substitutes $x_{1 \ldots 2}$ for the input disturbance $\varepsilon$,

$$
\begin{aligned}
& x=\left[\begin{array}{l}
x_{1} \\
x_{2}
\end{array}\right]=\left[\begin{array}{c}
\int \varepsilon d t \\
\varepsilon
\end{array}\right] \\
& \varepsilon=\Omega-\Omega_{r e q},
\end{aligned}
$$

the generator torque $Q_{g e n}$ as output of the PI-controller can be calculated,

$$
Q_{\text {gen }}=k_{p} \cdot \varepsilon+k_{I} \cdot \int \varepsilon=k_{p} \cdot x_{2}+k_{I} \cdot x_{1} .
$$

The rotor is a non-linear system and therefore requires linearization of the hydrodynamic torque $Q_{H y d r o}$ at the set point $\Omega_{0}$,

$$
Q_{\text {Hydro }}=\left.Q_{H y d r o}\right|_{\Omega_{0}}+\underbrace{\left(\Omega-\Omega_{0}\right)}_{=x_{2}} \cdot \frac{d Q_{H y d r o}}{d \Omega},
$$

with $\Omega_{0}=\Omega_{\text {req. }}=$ const here. With the momentum equation,

$$
\dot{\Omega}=\frac{1}{J} \cdot\left(Q_{\text {Hydro }}-Q_{\text {gen }}\right),
$$

the turbine behaviour can be concluded with the rotational inertia $J$,

$$
\dot{x}_{2}=\dot{\epsilon}=\dot{\Omega}-\dot{\Omega}_{r e q}=\dot{\Omega}=\frac{1}{J} \cdot\left(Q_{\text {Hydro }}-Q_{g e n}\right) .
$$

Summarizing this to the differential equation set,

$$
\begin{aligned}
& \dot{\boldsymbol{x}}=\boldsymbol{A}_{\boldsymbol{I}} \cdot \boldsymbol{x}+\boldsymbol{b}_{\boldsymbol{I}} \\
& {\left[\begin{array}{l}
\dot{x}_{1} \\
\dot{x}_{2}
\end{array}\right]=\underbrace{\left[\begin{array}{cc}
0 & 1 \\
-\frac{k_{I}}{J} & \frac{1}{J} \cdot\left(\frac{d Q_{H y d r o}}{d \Omega}-k_{p}\right)
\end{array}\right]}_{=\boldsymbol{A}_{\boldsymbol{I}}} \cdot\left[\begin{array}{l}
x_{1} \\
x_{2}
\end{array}\right]+\underbrace{\left[\begin{array}{c}
0 \\
\frac{\left.Q_{H y d r o}\right|_{\Omega_{0}}}{J}
\end{array}\right]}_{=\boldsymbol{b}_{\boldsymbol{I}}},}
\end{aligned}
$$

the poles of the inner loop $\lambda_{I_{1 / 2}}$ can be calculated

$$
\lambda_{I_{1 / 2}}=\operatorname{eig}\left(\boldsymbol{A}_{I}\right)=\frac{\left(\frac{d Q_{H y d r o}}{d \Omega}-k_{p}\right)}{2 \cdot J} \pm \sqrt{\left(\frac{\left(\frac{d Q_{H y d r o}}{d \Omega}-k_{p}\right)}{2 \cdot J}\right)^{2}-\frac{k_{I}}{J}}
$$

Therefore the stability limits of the inner loop are defined as,

$$
\begin{aligned}
& k_{I} \stackrel{!}{\geq} 0, \\
& k_{p} \stackrel{!}{>} \frac{d Q_{H y d r o}}{d \Omega} \forall \Omega \in\left[0, \Omega_{\max }\right] \wedge v_{1} \in\left[0, v_{1 \text { max }}\right] .
\end{aligned}
$$

However also the dynamic properties for the loop can be set and $k_{p}, k_{I}$ calculated from the damping ratio $\zeta$ and natural frequency $\omega_{n}$,

$$
\begin{aligned}
& k_{I}=\omega_{n}^{2} \cdot J \\
& k_{p}=2 J \cdot \sqrt{\frac{k_{I}}{J}} \cdot \zeta+\frac{d Q_{H y d r o}}{d \Omega} .
\end{aligned}
$$




\section{Outer Closed-Loop Stability}

On the results of the inner loop stability it can be concluded that the controller and the turbine operation is stable for the required speed being constant, $\dot{\Omega}_{r e q}=0$. Based on this finding, we can assume now that the inner loop is also stable with the change in required speed being sufficiently small, $\dot{\Omega}_{\text {req }} \rightarrow 0$ and subsequently the time constant of the outer loop being large enough. This assumption will be proven here and the stability analysis boils down to finding the fastest possible time constant of the outer loop.

Therefore, it can be deduced that the term $G_{Q_{g e n} \rightarrow \Omega_{\text {req }}}$ may consist of the characteristic curve $f(Q)$ of the controller combined with a $P T_{1}$-low pass filter element, as shown in Fig. 6 , and the controller is stable for $T_{P T_{1}}$ being sufficiently large. [11] and [12] used a more complex setup with PI controllers here, however this leaves issues on e.g. wind up and limits the options for adaptions in the characteristic curve.

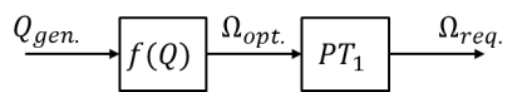

Fig. 6: $G_{Q_{g e n} \rightarrow \Omega_{\text {req }}}$

To determine the actual required value for $T_{P T_{1}}$ the dynamics of the overall loop need to be calculated. Substituting to the variables $z_{1 \ldots 4}$,

$$
z=\left[\begin{array}{c}
z_{1} \\
z_{2} \\
z_{3} \\
z_{4}
\end{array}\right]=\left[\begin{array}{c}
\int \Omega_{r e q} d t \\
\Omega_{r e q} \\
\int \Omega d t \\
\Omega
\end{array}\right],
$$

the PI-controller,

$$
Q_{\text {gen }}=k_{p} \cdot\left(z_{4}-z_{2}\right)+k_{I} \cdot\left(z_{3}-z_{1}\right) \text {, }
$$

and the linearized turbine behaviour, can be calculated as done for the inner loop.

The same linearization is done for $G_{Q_{g e n} \rightarrow \Omega_{r e q}}$ by linearizing the characteristic curve $f(Q)$,

$$
\begin{aligned}
& f(Q): \Omega_{\text {opt }}\left(Q_{\text {gen }}\right)=\min \left\{\begin{array}{l}
\frac{P_{\text {Rated }}}{Q_{\text {gen }}} \\
\sqrt{\frac{Q_{g e n}}{k}}
\end{array}\right. \\
& k=\frac{\rho \pi R_{\text {Rotor }}^{5} \cdot c_{p, o p t}}{2 \cdot \lambda_{\text {opt }}^{3}} \\
& \frac{d \Omega_{\text {opt }}}{d Q_{\text {gen. }}}=\left\{\begin{array}{lc}
-\frac{P_{\text {Rated }}}{Q_{\text {gen }}^{2}} & \forall \Omega_{\text {opt }}=\frac{P_{\text {Rated }}}{Q_{\text {gen }}} \\
\frac{1}{2 \cdot \sqrt{Q_{\text {gen }} \cdot k}} & \text { else }
\end{array}\right. \\
& \Omega_{\text {opt }}=\left.\Omega_{\text {opt }}\right|_{\Omega_{0}}+\left(Q_{\text {gen }}-\left.Q_{\text {gen }}\right|_{\Omega_{0}}\right) \cdot \frac{d \Omega_{\text {opt }}}{d Q_{\text {gen }}}
\end{aligned}
$$

and the $P T_{1}$-filter element,

$$
\dot{z}_{2}=\dot{\Omega}_{r e q}=\frac{1}{T_{P T_{1}}} \cdot\left(\Omega_{o p t}-\Omega_{r e q}\right)=\frac{\Omega_{o p t}-z_{2}}{T_{P T_{1}}} .
$$

These equations can be summarized to the differential equation of the overall system,

$$
\dot{\mathbf{z}}=\boldsymbol{A}_{\boldsymbol{o}} \cdot \boldsymbol{z}+\boldsymbol{b}_{\boldsymbol{o}}
$$

$$
\begin{aligned}
& {\left[\begin{array}{c}
\dot{z}_{1} \\
\dot{z}_{2} \\
\dot{z}_{3} \\
\dot{z}_{4}
\end{array}\right]=\underbrace{\left[\begin{array}{cccc}
0 & 1 & 0 & 0 \\
-\frac{k_{I}}{T_{P T_{1}}} \cdot \frac{d \Omega_{o p t}}{d Q_{g e n}} & \frac{-k_{p} \cdot \frac{d \Omega_{o p t}}{d Q_{g e n}-1}}{T_{P T_{1}}} & \frac{k_{I}}{T_{P T_{1}}} \cdot \frac{d \Omega_{\text {opt }}}{d Q_{g e n}} & \frac{k_{p}}{T_{P T_{1}}} \cdot \frac{d \Omega_{\text {opt }}}{d Q_{g e n}} \\
0 & 0 & 0 & 1 \\
\frac{k_{I}}{J} & \frac{k_{p}}{J} & -\frac{k_{I}}{J} & \frac{\frac{d Q_{H y d r o}}{d \Omega}-k_{p}}{J}
\end{array}\right]}_{=A_{O}} \cdot\left[\begin{array}{l}
Z_{1} \\
z_{2} \\
z_{3} \\
z_{4}
\end{array}\right]+} \\
& \underbrace{\left[\begin{array}{c}
0 \\
\Omega_{\text {soll }} \Omega_{\Omega_{0}}-\left.Q_{\text {gen }}\right|_{\Omega_{0}} \cdot \frac{d \Omega_{\text {opt }}}{d Q_{g e n}} \\
T_{P T_{1}} \\
0 \\
\frac{\left.Q_{H y d r o}\right|_{\Omega_{0}}-\Omega_{0} \cdot \frac{d Q_{H y d r o}}{d \Omega}}{J}
\end{array}\right]}_{=\boldsymbol{b}_{\boldsymbol{O}}}
\end{aligned}
$$

The analytical solution for the eigenvalues $\lambda_{o}$ of $\boldsymbol{A}_{\boldsymbol{O}}$ is complex and not done here. Instead a numerical solution is suggested and used to solve for the value of $T_{P T_{1}}$ required for stability as shown below.

It needs to be mentioned that 1 eigenvalue is 0 independent from the parameters, indicating that the set of variables $z_{1 \ldots 4}$ could be condensed to a 3 dimensional set. However this would increase the complexity of the matrix $\boldsymbol{A}_{\boldsymbol{o}}$.

\section{E. Dynamic Behaviour}

As indicated above, the system matrix $\boldsymbol{A}_{\boldsymbol{O}}$ is solved here with a numerical solver. Fig. 7 shows the exemplary results of this procedure for an arbitrary point in the above rated region $\left(v_{1}>v_{\text {rated }}\right)$ of the turbine with $k_{P}=1.1 e 7 \mathrm{sNm}$, $k_{I}=1 e 8 \mathrm{kgm}^{2} \mathrm{~s}^{-2} \quad, \quad$ resulting in $d \Omega_{\text {opt }} / d Q_{\text {gen }}=$ $-1.45 e 6(\mathrm{sNm})^{-1}$ and $d Q_{H y d r o} / d \Omega=96.3 e 3 \mathrm{sNm}$.

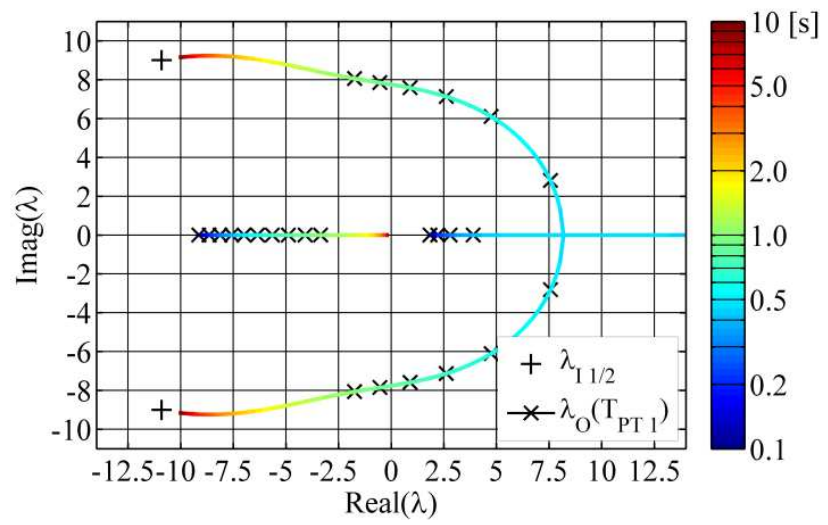

Fig. 7: Pole location of $\boldsymbol{A}_{\boldsymbol{O}}$ coloured by $T_{P T_{1}}$ for an arbitrary point of operation above rated

While the first eigenvalue of $\boldsymbol{A}_{\boldsymbol{O}}$ is real and negative for all values of $T_{P T_{1}}$, the other poles form a pair whitch is positive real for small values of $T_{P T_{1}}$. In this case the system is unstable. With increasing $T_{P T_{1}}$ the pole pair gets an imaginary part and converges to the poles of the inner loop $\lambda_{I 1 / 2}$ for $T_{P T_{1}} \rightarrow \infty$. This confirms the initial analysis of the stability $-\mathrm{a}$ sufficiently slow change of $\Omega_{r e q}$ resp. a sufficiently large $T_{P T_{1}}$ results in a stable operation of the underspeed controller.

In Fig. 8 the required value of $T_{P T_{1}}$ is shown. For below rated operation the control loop is stable independent of $T_{P T_{1}}$. In this case the $P T_{1}$ element could therefore be neglected. 
However, for above rated operation the required value spikes near the rated point due to the required fast deceleration of the rotor to maintain $P=P_{\text {rated }}$, cf. Fig. 3. Beyond this large gradient in the curve of steady points of operation a value of $T_{P T_{1}} \approx 1 s$ leads to a stable operation.

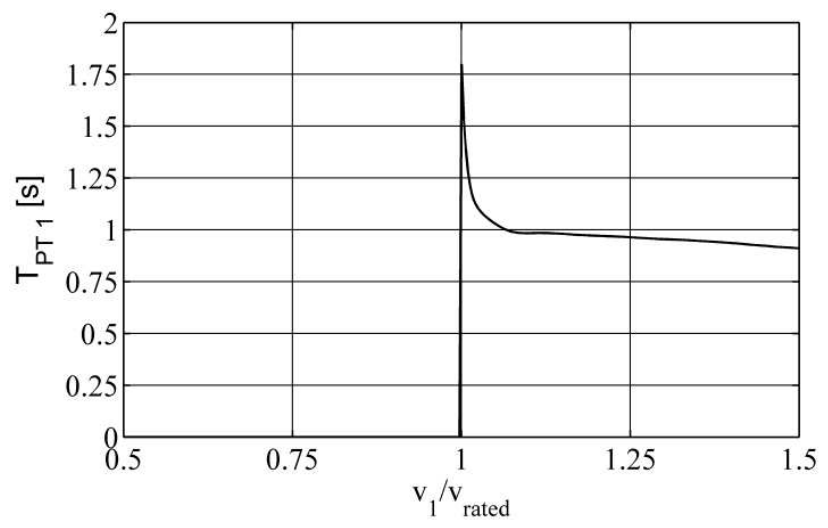

Fig. 8: Required value of $T_{P T_{1}}$ for stable operation

As a result of the pole locations shown in Fig. 7 the optimal value of $T_{P T_{1}}$ is a compromise of the fast reaction of the pole on the real axis and the damping ratio of the imaginary pole pair. As shown in Fig. 9 for a step change in current velocity a smaller value of $T_{P T_{1}}$ results in oscillations of the speed $\Omega$ and the electrical power $P$ including a high initial peak in power output. On the other hand a larger value decreases the power peak, however it takes a long time for the turbine to return to the rated power. Therefore an average value should be chosen, resulting in a reduced peak and fast return to the desired power levels. For the given parameters $T_{P T_{1}}=1.5 \mathrm{~s}$ fulfills these requirements.

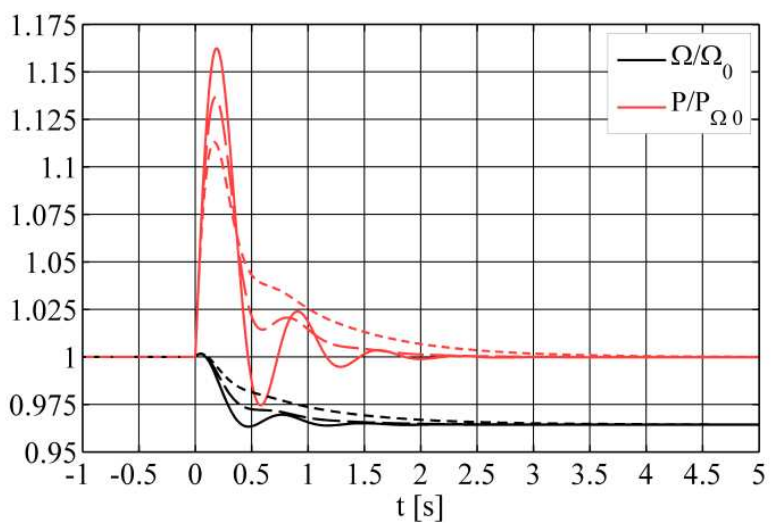

Fig. 9: Step response to increase of current speed $v_{1}=3.5 \rightarrow 3.6 \mathrm{~m} / \mathrm{s}$ for $T_{P T_{1}}=1.25 \mathrm{~s}$ (solid), $T_{P T_{1}}=1.5 \mathrm{~s}$ (dashed) and $T_{P T_{1}}=2 \mathrm{~s}$ (dotted)

\section{SIMULATION ENVIRONMENT}

To analyse the behaviour of the underspeed controller and compare it to state of the art approaches a non-linear model has been setup and implemented in Matlab Simulink.

\section{A. Dynamic Model}

Based on the results of [3] the most significant impact to turbine loads are the nacelle motion $\Delta x$ and obviously the rotor speed $\Omega$. For the stability analysis above only a linearized model of the rotor speed $\Omega$ has been taken into account. However, to analyse the dynamic loadings, the structural model is extended to the non-linear setup taking the 2 degrees of freedom of nacelle motion $\Delta x$ and the rotor speed $\Omega$ into account,

$$
w=\left[\begin{array}{l}
w_{1} \\
w_{2} \\
w_{3}
\end{array}\right]=\left[\begin{array}{c}
\Omega \\
\Delta x \\
\Delta \dot{x}
\end{array}\right] .
$$

Assuming a pure axial motion of the nacelle and rotor, nullifying the gyroscopic effects, the equation of motion for the tower DoF can be simplified to a harmonic oscillator

$$
m_{\text {Head }}^{\star} \cdot \Delta \ddot{x}+c \cdot \Delta x=F_{H y d r o}(\Delta \dot{x}) \text {. }
$$

Here $m_{\text {Head }}^{\star}$ is the sum of head mass $m_{\text {Head }}$ and added mass with the added mass coefficient $C_{a}$,

$$
m_{\text {Head }}^{\star}=m_{\text {Head }}+\rho \cdot V_{\text {Nacelle }} \cdot C_{a} \text {. }
$$

With the equation of motion for the rotor DoF as shown above, cf. Eq. (6), the dynamic model of the turbine can be concluded as

$$
\left[\begin{array}{c}
\dot{\Omega} \\
\Delta \dot{x} \\
\Delta \ddot{x}
\end{array}\right]=\left[\begin{array}{c}
\frac{Q_{\text {Hydro }}(w)}{J} \\
W_{3} \\
\frac{F_{\text {Hydro }}(w)-c \cdot \Delta x}{m_{\text {Head }}^{\star}}
\end{array}\right]+\left[\begin{array}{c}
-\frac{Q_{g e n}}{J} \\
0 \\
0
\end{array}\right],
$$

with the system input $Q_{g e n .} . F_{H y d r o}$ is calculated as the sum of the Morison equation and the rotor thrust $T_{H y d r o}$,

$$
\begin{aligned}
F_{\text {Hydro }}= & T_{\text {Hydro }}\left(v_{1}+\Delta \dot{x}\right)+\underbrace{\frac{d v_{1}^{\star}}{d t} \cdot \rho V_{\text {Nacelle }} \cdot\left(C_{a}+1\right)}_{\text {Froude }- \text { Krylov }}+ \\
& \underbrace{\frac{\rho}{2} \cdot A \cdot c_{d} \cdot\left(v_{1}^{\star}-\Delta \dot{x}\right) \cdot\left|v_{1}^{\star}-\Delta \dot{x}\right|}_{\text {rrag }} .
\end{aligned}
$$

Incorporating the findings of [5] the nacelle is however too large for being subject to the local and instantaneous current velocity $v_{1}$, but is averaging the current field by its size to $v_{1}^{\star}$. This was taken into account by applying a low pass filter $\left(P T_{1}\right)$ to the velocity with the time constant being equal to passage time of a particle along the nacelle length $l_{\text {Head }}=13 \mathrm{~m}$,

$$
v_{1}^{\star}=\left.P T_{1}\left(v_{1}\right)\right|_{T_{1}=\frac{v_{1}}{l_{\text {Head }}}} \text {. }
$$

Also to account for the dynamics of the power electronics an additional time constant of $T_{\text {Geno }}=0.02 \mathrm{~s}$ is added between $T_{\text {geno }}$ applied to the rotor DoF and the controller output value.

\section{B. Hydrodynamic Representation}

For each set of mean current velocity $\bar{v}_{1}$ and turbulence intensity Tu 6 random seeds of turbulent velocity have been defined and generated with the TurbSim, [14], 'tidal' spectral model. As TurbSim has a prescribed turbulence intensity implemented for the 'tidal' spectral model, the hub-height velocity is then scaled to the desired Tu. Each seed has a length of $700 s$ for an effective simulation time of $600 s$ excluding the onset. The results of the 6 seeds are merged to the mean, min resp. max value during all runs and to the mean value of the 6 standard deviations $\sigma$ resp. damage equivalent 
loads DEL. The later is calculated with the rainflow algorithm, [15], for $N_{\text {ref }}=2 \cdot 10^{6}$ and the Wöhler exponent of $m=4$ (steel).

As shown above the nacelle hydrodynamics are subject to an additional low pass filtering, however, for the rotor hydrodynamics the rotor performance and load is calculated directly from the instantaneous velocity $v_{1}$ without taking additional dynamics into account,

$$
\begin{aligned}
& Q_{\text {Hydro }}=\frac{\rho}{2} \pi R_{\text {Rotor }}^{3} \cdot v_{1}^{2} \cdot c_{Q}\left(\lambda_{T S R}, \beta\right), \\
& T_{\text {Hydro }}=\frac{\rho}{2} \pi R_{\text {Rotor }}^{2} \cdot v_{1}^{2} \cdot c_{T h}\left(\lambda_{T S R}, \beta\right) .
\end{aligned}
$$

This distinction is necessary due to the difference in size and transient behaviour of the components.

\section{Simulation Model}

The simulation model is setup in Simulink based on the block diagrams used for the controller development above. However, as a non-linear turbine model is used for the load simulations it was replaced with an implementation of the dynamic model described above and sketched in Fig. 10.

To achieve a good balance of computational efficiency and accuracy the 'ode2' method, a $2^{\text {nd }}$ order fixed step solver, with a time step of $d t=0.002 \mathrm{~s}$ was chosen. This value is at least 1 order of magnitude smaller than any frequency of the system. The other solver methods implemented offered only minor increased accuracy with significantly higher resources.

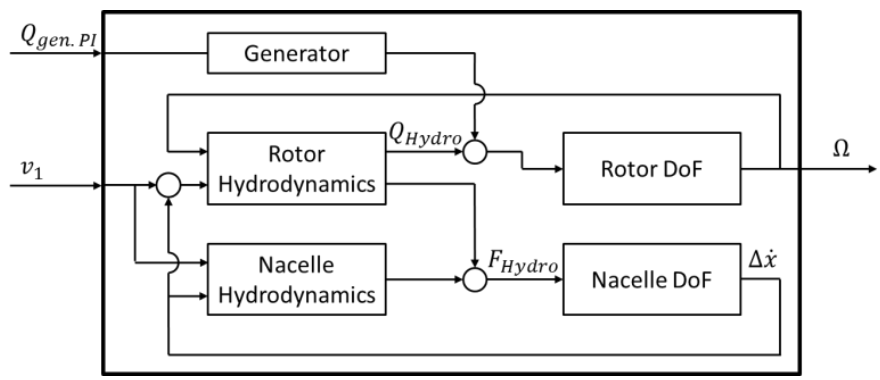

Fig. 10: Block diagram of turbine model

\section{COMPARISON OF CONTROL STRATEGIES TO STATE OF THE ART CONTROLLERS}

Within this section the controller will be evaluated and compared to state of the art controllers.

\section{A. Controller Optimisation}

As shown above, cf. Fig. 3, maintaining the rated power output of the turbine requires a steep change of $\Omega$ with increasing current velocity $v_{1}$. A current velocity oscillating around the rated velocity would result in fast fluctuations in rotor speed and subsequent the power output would be highly volatile. This was also indicated by the required large controller time constant $T_{P T_{1}}$ in this region, cf. Fig. 8. It is therefore desired to reduce this impact by adapting the characteristic curve $f(Q): \Omega_{\text {opt }}\left(Q_{\text {gen }}\right)$.

In Fig. 11 two options for modification are shown. On the one hand a clip of the speed, on the other a smooth transition of the above and below rated region with a $4^{\text {th }}$ order polynomial curve. Both options reduce the spike in the $T_{P T_{1}}$ curve significantly to $\max \left(T_{P T_{1}}\right) \approx 1.3 \mathrm{~s}$ with clipping resp. $1.2 \mathrm{~s}$ for the polynomial curve. Based on these values, $T_{P T_{1}}$ was chosen to be $2 s$ for the original curve and $1.5 s$ for both others.

Fig. 12 shows the power output of the turbine for each case. As it can be seen, the modifications resulted in a significant increase in power quality, resp. decrease of standard deviation of electrical power $\sigma\left(P_{e l}\right)$, while sacrificing an acceptable small portion of the mean power output $\bar{P}_{e l}$. The highest loss in power output occurs at $v_{1}=v_{\text {rated }}$ with $\Delta \bar{P}_{e l} \approx 1.3 \%$ resulting in a loss of annual energy production AEP of $0.72 \%$ assuming a site with $v_{1}=1.3 \cdot v_{\text {rated }} \cdot \sin \left(2 \pi \cdot t / T_{\text {Tide }}\right)$. As the clipped characteristic curve features lower peak power the further simulations are done with the clipped characteristic curve and $T_{P T_{1}}=1.5 \mathrm{~s}$.

The same issue of high power fluctuations at rated current speed was also observed by [17]. However as mentioned above, [17] used a PI-Controller for the turbine power and therefore did not have the option to shape the characteristic curve, but needed to shape the power over current speed curve. It therefore needed to rely on a theoretic option to have the momentary current speed $v_{1}$ measured online to deal with the issue. As discussed, for the here presented controller setup no additional sensor inputs are required.

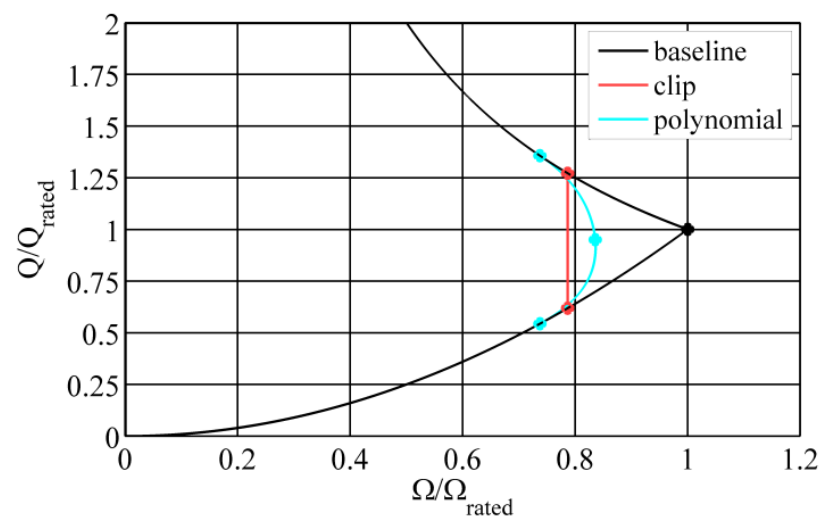

Fig. 11: Modified characteristic curves $f(Q): \Omega_{\text {opt }}\left(Q_{\text {gen }}\right)$

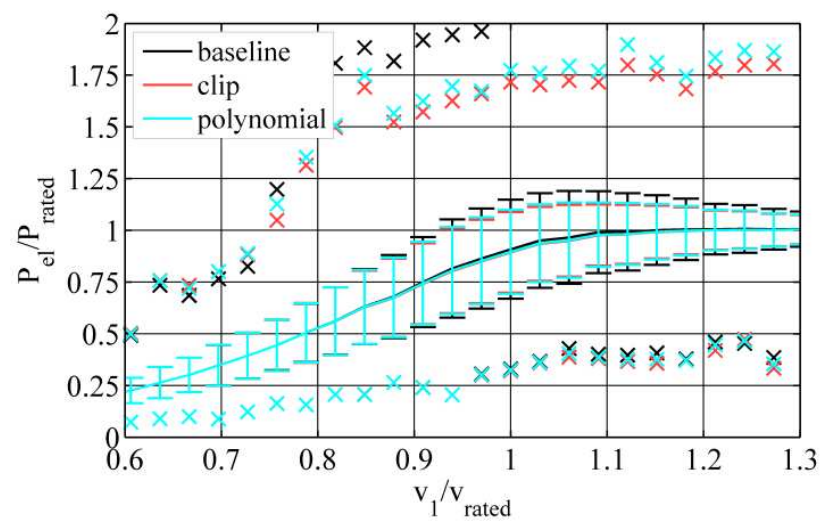

Fig. 12: Mean power output $\bar{P}_{e l}$ (solid), standard deviation $\pm \sigma\left(P_{e l}\right)$ (error bars) and peak power (x) for turbine with $\mathrm{Tu}=10 \%$ turbulence intensity 


\section{B. Baseline Controllers}

For comparison to the state of the art, two baseline controllers, an overspeed control and a pitch to feather concept, are defined. The overspeed control as described above applies the generator torque $Q_{\text {gen.os }}$ accordingly to the rotor speed $\Omega$,

$$
Q_{\text {gen } 0 S}(\Omega)=\min \left\{\begin{array}{l}
\frac{P_{\text {Rated }}}{\Omega} \\
k \cdot \Omega^{2}
\end{array} .\right.
$$

The pitch control is based on the controller described by [13] for the NREL 5MW baseline wind turbine. However, several options typical for wind turbines, as keeping the rotor speed constant with $v_{1}$ slightly smaller than rated current $v_{\text {rated }}$ (region 2.5 ) and a hysteresis in the torque curve,

$$
Q_{\text {gen Pitch,Literature }}(\Omega)=\left\{\begin{array}{cc}
Q_{\text {gen os }}(\Omega) & \Theta<1^{\circ} \\
\frac{P_{\text {Rated }}}{\Omega} & \text { else }
\end{array},\right.
$$

proved to be problematic with the low inertia of the tidal turbine. Therefore the generator curve of the overspeed control is also used for the pitch controlled turbine here with the target speed for the pitch angle controller being $\Omega_{\text {pitch }}=$ $\left.1.05 \cdot \Omega_{\mathrm{OS}}\right|_{v=v_{\text {rated }}}$. The pitch angle $\Theta$ is controlled by a gain scheduled PI-controller,

$$
\begin{aligned}
& K_{P \Theta}(\Theta)=\frac{2 J \Omega_{\text {rated }} \zeta_{\varphi} \omega_{\varphi n}}{-\left.\frac{\partial P}{\partial \Theta}\right|_{\Theta=0^{\circ}}} \cdot G K(\Theta) \\
& K_{I \Theta}(\Theta)=\frac{J \Omega_{\text {rated }} \omega_{\varphi n}^{2}}{-\left.\frac{\partial P}{\partial \Theta}\right|_{\Theta=0^{\circ}}} \cdot G K(\Theta) .
\end{aligned}
$$

The gain scheduler $G K(\Theta)$ is based on the actual pitch angle $\Theta$,

$$
G K(\Theta)=\frac{1}{1+\frac{\Theta}{\Theta_{K}}},
$$

and the reference pitch angle $\Theta_{K}$ with double the sensitivity $\partial P / \partial \Theta$ of $\Theta=0^{\circ}$,

$$
\Theta_{K}=\Theta\left(\frac{\partial P}{\partial \Theta}=\left.2 \cdot \frac{\partial P}{\partial \Theta}\right|_{\Theta=0^{\circ}}\right) \text {. }
$$

[13] suggests the response characteristics to be $\omega_{\varphi n}=$ $0.6 \mathrm{rad} / \mathrm{s}, \zeta_{\varphi}=0.7$, however they need to be adapted. For the present tidal turbine $k_{P \Theta}=0.9 \mathrm{~s} \cdot G K(\Theta)$ and $T_{I \Theta}=$ $k_{P \Theta} / k_{I \Theta}=1.5 \mathrm{~s}$ are found to be suitable. The pitch rate is limited to $|d \Theta / d t| \leq 10^{\circ} / \mathrm{s}$.

\section{Turbine Loads and Power Output}

The main purpose of developing the underspeed controller is to reduce the turbine loads during operation. As shown in Fig. 13, in the comparison of the different controller approaches this aim is achieved. Despite the rotor blade investigated here was optimised for overspeed operation, [4], the average loads are significantly reduced. With the pitch controller it was possible to further reduce the average thrust, but the DELs are by a factor of 2 smaller for the underspeed controller. The same applies for the peak load amplitude with the best performance on the underspeed controller.

Fig. 3 indicated a reduction in thrust based on the steady points of operation, which is no more present in the turbulent cases shown here. As shown by Fig. 14 this is an impact of the turbulence intensity. The mean thrust is reduced around the rated point with increasing turbulence intensity, but increases for higher current speeds. In the same way also the DELs and peak loads are increasing with turbulence intensity, as expected.

The same analysis has also been done including the tower shadow assuming downstream operation. The impact to the rotor was therefore approximated from the results of [2] with a Fourier series resulting in a strong $3 \Omega$ and $6 \Omega$ excitation of the tower. However the qualitative results didn't change compared to upstream operation, so this is not detailed here.

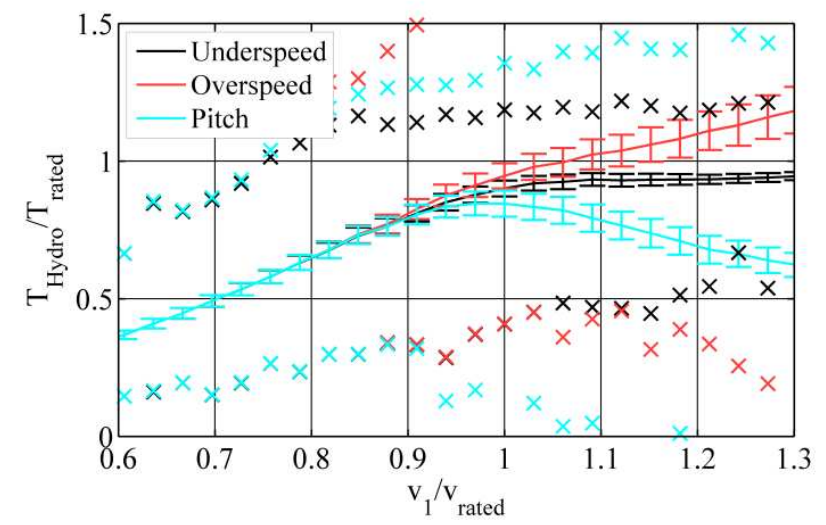

Fig. 13: Mean rotor thrust $\bar{T}_{\text {Hydro }}$ (solid), damage equivalent load $\pm D E L\left(T_{\text {Hydro }}\right)$ (error bars) and peak loading (x) for turbine with $T u=10 \%$ turbulence intensity

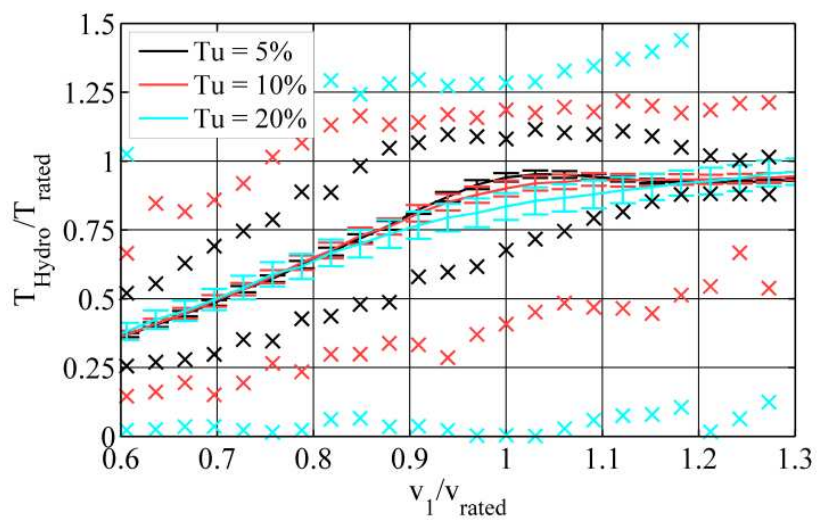

Fig. 14: Mean rotor thrust $\bar{T}_{\text {Hydro }}$ (solid), damage equivalent load $\pm D E L\left(T_{\text {Hydro }}\right)$ (error bars) and peak loading (x) for turbine with underspeed controller

For the rotor torque this comparison of the controllers changes, see Fig. 15. The underspeed controller causes now higher torque loadings than both other approaches. However, this was to be expected as the concept of the underspeed controller relies on an increase in torque. More interesting is here, that also the DELs of the torque are reduced by the underspeed control compared to the other approaches for high current speeds.

On the power output, Fig. 16, all controllers proofed to be able to maintain rated power. However as the point of operation for the underspeed controller is unstable by nature and stabilized by the controller, the peak power output is 
increased. The best power quality was given by the overspeed controller.

From the analysis of the loads it can be concluded, that the underspeed controller is able to reduce the (thrust) loads of the tidal current turbine, however on the cost of additional loads in the drivetrain.

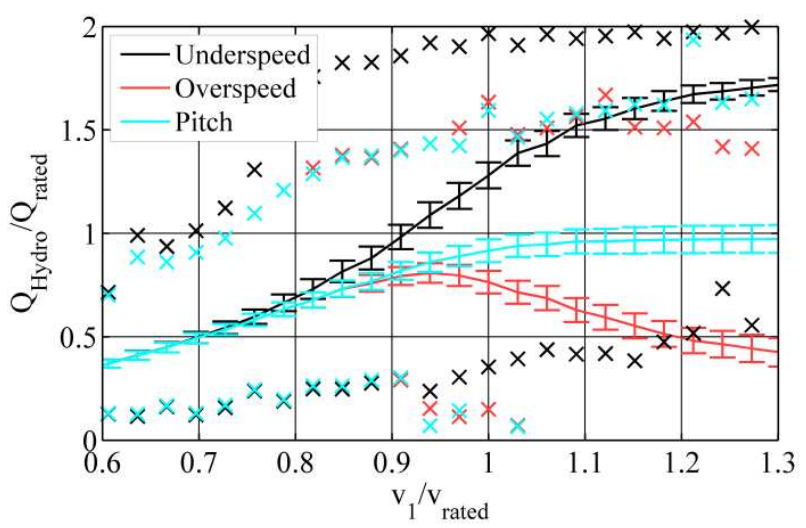

Fig. 15: Mean rotor torque $\bar{Q}_{H y d r o}$ (solid), damage equivalent load $\pm D E L\left(Q_{H y d r o}\right)$ (error bars) and peak loading (x) for turbine with $T u=10 \%$ turbulence intensity

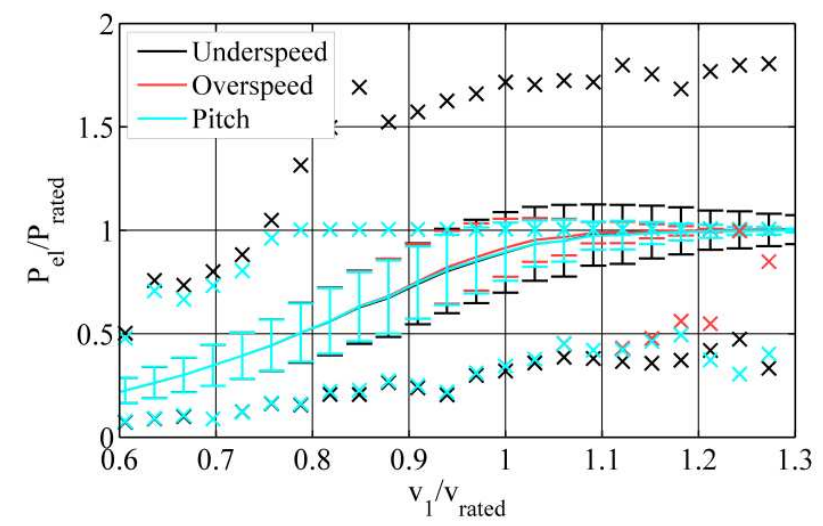

Fig. 16: Mean power output $\bar{P}_{e l}$ (solid), standard deviation $\pm \sigma\left(P_{e l}\right)$ (error bars) and peak power (x) for turbine with $T u=10 \%$ turbulence intensity

\section{IMPACTS TO TURBINE DESIGN AND CHARACTERISTICS}

To achieve an optimal system, the turbine and the controller cannot be designed independently. Above, the turbine was assumed to be a predefined unit and the controller was added. In this section some key issues of the underspeed controller and the changes in the turbine concept are discussed required to optimal fit the controller. These cover the power system, the hydrofoils and the blade design.

\section{A. Power System}

The first discussed issue of the underspeed controller is the volatile power output. This is the result of a trade-off for the fluctuating power in the current between the structural loads, the system complexity and an electrical smooth power output. The underspeed controller places this balance on the electrical system, which has to deal with the fluctuations.

For the generator it is unavoidable to design it to withstand very short but intense power peaks. As shown on the example case in Fig. 12 these peaks might even reach up to double rated power. However as the average electrical power is approx. at rated, this is mainly a question of the thermal capacity of the generator.

For the volatile power on the grid entry point, there are 3 basic ideas of how to deal with it; burning the peak power, smooth it on a turbine level or smooth it on the grid level. An initial analysis of each option is given here.

The first option, burning the peak power, can be implemented e.g. by short circuiting the generator with an active crowbar system for a time span long enough to reestablish the required rotor speed and rated power. However as we want to produce energy and not waste it this option is not preferable. Also the generator needs to be suitable for this type of operation.

Using the grid on the other hand for smoothing the power output, requires a grid strong enough to maintain stable operation with fluctuating power input, which might be but is not always given. Also the inverter needs to be designed to withstand the full peak power and will therefore be expensive subsequently.

This leaves the option of smoothing the power on the turbine level. The inverter typically consists of a generatorside converter and a grid-side converter coupled with a DClink. Adding a storage capacity $C_{D C}$ to the DC-link the power output can be levelled. Assuming the power at the grid entry point $P_{\text {grid }}$ may be $5 \%$ above $P_{\text {rated }}$ and is coupled to the stored energy $0 \leq E_{D C} \leq C_{D C}$,

$$
\begin{aligned}
& P_{\text {grid }} \leq P_{\text {rated }} \cdot\left(1+5 \% \cdot \frac{E_{D C}}{C_{D C}}\right), \\
& E_{D C}=\int P_{e l}-P_{\text {grid }} d t,
\end{aligned}
$$

the required capacity $C_{D C}$ can be calculated. In all cases simulated in this research a capacity of $C_{D C}=1 M W S=$ $0.28 \mathrm{kWh}$ was well large enough to result in a smooth power output limited to $105 \% P_{\text {rated }}$, Fig. 17.

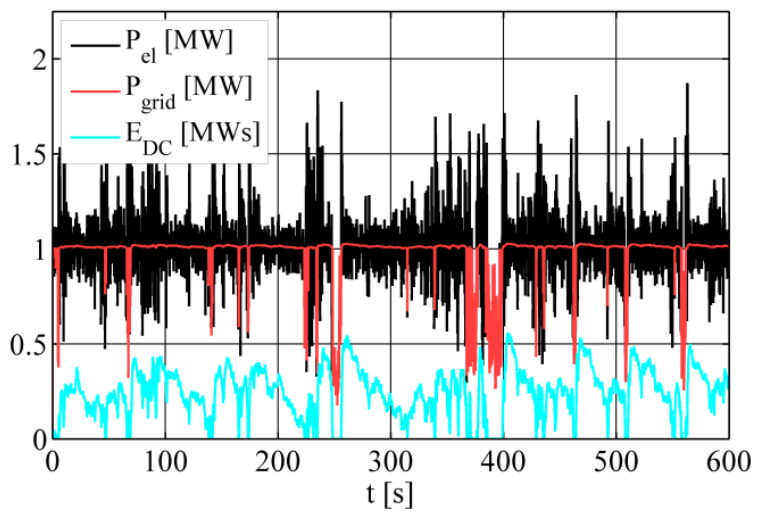

Fig. 17: Power at grid entry point $P_{\text {grid }}$ for underspeed controlled turbine with capacity in DC-link, $v_{1}=4.5 \frac{\mathrm{m}}{\mathrm{s}}$ current speed and $T u=10 \%$ turbulence intensity

\section{B. Hydrofoil stall}

Another type of issue is the stall on the hydrofoil. As this is the key element for the functionality of the controller it needs to be taken into account during design. By nature stall is an 
unsteady and transient effect which will cause fluctuating lift and drag forces on the rotor blade. To evaluate these for the present example case of the HyTide ${ }^{\circledR} 1000-13$ rotor blade a CFD simulation has been carried out using a steady inflow, Fig. 18.

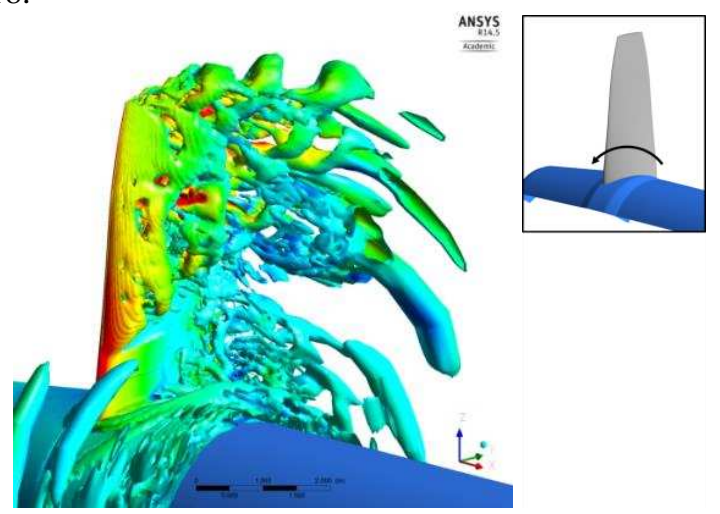

Fig. 18: Stall structure in wake of rotor blade with $\lambda_{T S R}=\lambda_{\text {opt }} / 2$ coloured by velocity

The model is based on the setup of [1], but has been limited to a $120^{\circ}$ periodic case and the grid has been refined to a high quality grid with approx. $3.6 \cdot 10^{6}$ structured elements. Further the turbulence model has been changed to the SAS SST model as this one is more appropriate for stall simulations than the classic SST model, [16].

Fig. 19 shows the thrust coefficient $c_{T h}$ and power coefficient $c_{P}$ over time for a point of operation at $\lambda_{T S R}=$ $\lambda_{\text {opt }} / 2$. As can be seen, both values are fluctuating stochastically causing additional loads on the turbine. However, a more detailed analysis of this case revealed that the origin for these fluctuations is concentrated on the outer radii, Fig. 20.

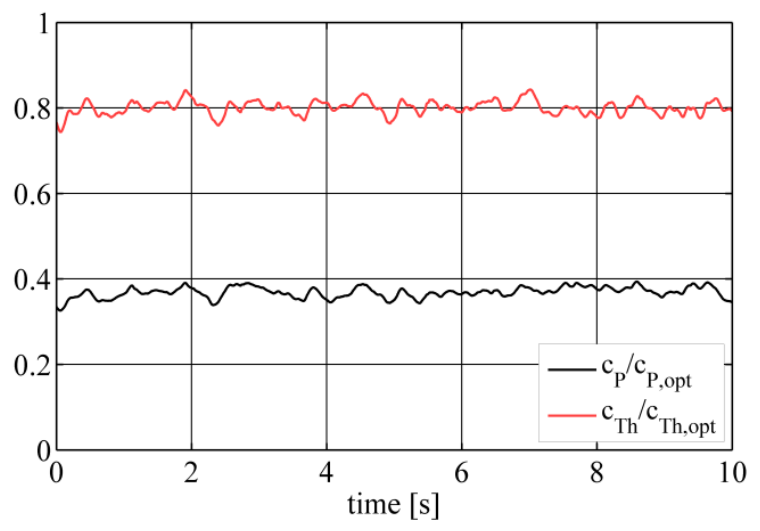

Fig. 19: Thrust, $c_{T h}$, and power, $c_{P}$, coefficient for HyTide ${ }^{\circledR} 1000-13$ rotor blade with $\lambda_{T S R}=\lambda_{\text {opt }} / 2$

The comparison to Fig. 18 indicates that the hydrofoils used in inner and outer section differ in their stall behaviour and cause this localisation. While the inner hydrofoils are thick and have trailing edge stall behaviour, the outer are thin with leading edge stall, optimised for overspeed controlled operation. As shown by [7] it is a systematic behaviour that leading edge stall causes unsteady currents and load fluctuations while trailing edge stall is more stable.
Therefore it is desired to choose trailing edge stall hydrofoils for an underspeed operated turbine. The hydrofoil should not have a suction peak as this would cause leading edge stall. Typically this is geometrically indicated by a large leading edge radius and thickness.

By choosing the appropriate type of hydrofoil the issue of unsteady stall can therefore be accepted. Also it has to be mentioned that this is an investigation for a homogenous inflow. In case of a turbulent, unsteady inflow the stall patterns will further change and be less stochastic but following the apparent turbulence.

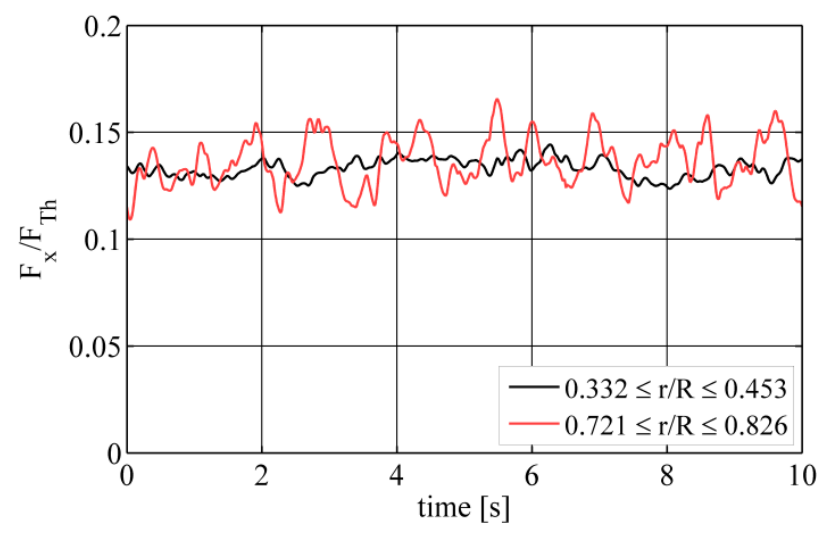

Fig. 20: Thrust force $F_{x}$ integrated for 2 radial sections

\section{Blade Design Targets}

The rotor blade has the largest impact to the turbine design. By adapting this component to the control strategy optimal system behaviour can be achieved. In general, this means for a tidal current turbine that optimal power production with limited loads in below rated and minimal loads with constant rated power output in above rated operation is desired. Taking the underspeed control into account we can conclude for the design target:

(i) $\quad c_{P}=c_{p, o p t} \uparrow \forall \lambda_{T S R}=\lambda_{\text {opt }} \pm d \lambda_{\text {opt }}$

(ii) $\quad c_{P} \sim \lambda^{3} \forall \lambda_{T S R} \leq \lambda_{\text {opt }}-d \lambda_{\text {opt }}$

(iii) $\quad c_{P} / c_{T h} \uparrow$

Among these (iii) is the most important as it governs the loads. However, to achieve the design targets there are only 3 parameters for each section of the rotor blade - chord length $t_{\text {chord }}$, twist angle $\alpha_{\text {twist }}$ and the hydrofoil. Based on these parameters the characteristics of the rotor blade have to be shaped for optimal operation in a trade-off between the design targets.

With design target (i) we can deduce that no stall should occur within $\lambda_{\text {TSR }}=\lambda_{\text {opt }} \pm d \lambda_{\text {opt }}$. The magnitude of $d \lambda_{\text {opt }}$ can be calculated from the maximum gust intensity $d v_{1}$, e.g. $0.2 \cdot v_{1}$, resulting in the required stall margin $d \alpha_{A o A}$,

$$
\begin{aligned}
& d \alpha_{A o A}=\alpha_{\text {design }}-\alpha_{\text {stall }}=\alpha_{2}-\alpha_{2,+d v_{1}}, \\
& \alpha_{2,+d v_{1}}=\operatorname{atan}\left(\frac{\left(v_{1}+d v_{1}\right) \cdot(1-a)}{\Omega \cdot r}\right) \text { with } \approx \text { const } .
\end{aligned}
$$

Therefore for each radial station $r$ the maximum design angle of attack $\alpha_{A o A}=\alpha_{\text {design }}$ can be deduced, 


$$
\alpha_{\text {design }}=\alpha_{\text {stall }}-\operatorname{atan}\left(\frac{\left(1+\frac{d v_{1}}{v_{1}}\right) \cdot(1-a)}{\lambda_{\text {opt }} \cdot\left(\frac{r}{R}\right)}\right)+\operatorname{atan}\left(\frac{1-a}{\lambda_{\text {opt }} \cdot\left(\frac{r}{R}\right)}\right)
$$

Ideally this value should coincide with the maximum glide ratio $\alpha_{A o A}\left(\varepsilon_{o p t}\right)$ based on the hydrofoil design to result in optimal power production and the design point being the optimal point $\lambda_{\text {opt }}=\lambda_{\text {design }}$.

The more constant the value $d v_{1}$ is along the radius, the sharper the transition between the plateau in the $c_{P}\left(\lambda_{T S R}\right)$ curve, design target (i), and the stalled region, design target (ii). However, as the regions can't be shaped independently the radial spread of this parameter needs to be optimised to meet the design targets depending on the actual hydrofoil characteristics.

Design target (iii) can be translated to 2 operational requirements: On one hand for the hydrofoil a high glide ratio $\varepsilon=c_{l} / c_{d}$ is required for the full range of operation. This results in a hydrofoil with high value $\varepsilon$ in a wide range of angles of attack $\alpha_{A o A}$ and subsequent a smooth stall behaviour. Typically this correlates with trailing edge stall hydrofoils as already requested by the stall analysis.

On the other hand for the optimal design point (iii) translates into a reduced induction ratio $a$ at the design point. Reducing this value too far would jeopardize (i) resulting again in a trade-off between targets. A value of e.g. $a=0.25$ compared to the Betz optimum $a=0.333$ is suitable. Therefore including the results of the stall margin, the twist angle $\alpha_{\text {twist }}$ and chord length $t_{\text {chord }}$ can be calculated from a modified Betz blade design,

$$
\begin{aligned}
& c_{\text {Th,Momentum }}(a)=4 a \cdot(1-a), \\
& t_{\text {chord }}(r) \approx \frac{2 \pi R \cdot c_{T h, \text { Momentum }}}{n_{\text {blades }} \cdot c_{l}\left(\alpha_{\text {AoA,design }}\right) \cdot \lambda_{\text {opt }} \cdot \sqrt{\lambda_{\text {opt }}^{2} \cdot\left(\frac{r}{R}\right)^{2}+\frac{4}{9}}}, \\
& \alpha_{\text {twist }}(r)=\operatorname{atan}\left(\frac{1-a}{\lambda_{\text {opt }} \cdot\left(\frac{r}{R}\right)}\right)-\alpha_{\text {AoA,design }} .
\end{aligned}
$$

With the above given set of equations a rotor blade suitable for underspeed operation can be designed. Further optimisation might be necessary but requires a more detailed analysis of the turbine system and operating conditions.

\section{CONCLUSIONS}

The present paper introduced a control method for fixed pitch tidal current turbines based on a variable speed system forcing the rotor into stall - the underspeed control. After discussing the stability, the paper compared the performance of the underspeed controller to state of the art control techniques.

The underspeed method was able to reduce the fatigue damage equivalent rotor loads (thrust) as well as the ultimate loads significantly. Compared to a baseline pitch controller the DELs are reduced by a factor of 2 and a factor of 3 compared to the overspeed controller. Although also the drivetrain DELs were reduced slightly, this load reduction was on the cost of increasing mean drivetrain loads.
The paper concluded with an outlook to the design impacts of the controller to the turbine system and showed the suitability to future use on tidal current turbines.

\section{ACKNOWLEDGMENT}

This research has been conducted within a joint project of Stuttgart Wind Energy, University Stuttgart, and Voith Hydro Ocean Current Technologies $\mathrm{GmbH} \&$ Co. KG. Further it is supported by the software suppliers Simpack AG and Ansys Germany GmbH.

\section{REFERENCES}

[1] M. Arnold, F. Biskup, D. Matha "Untersuchung einer Gezeitenströmungsturbine und Haltestruktur mittels strömungsmechanischer Simulation“, thesis, University Stuttgart, Germany, Okt. 2011

[2] M. Arnold, F. Biskup, P.W. Cheng "Simulation of Rotor-FoundationInteraction on Tidal Current Turbines with Computational Fluid Dynamics", $10^{\text {th }}$ EWTEC 2013, Aalborg, Denmark

[3] M. Arnold, F. Biskup, P.W. Cheng "Impact of Structural Flexibility on Loads on Tidal Current Turbines", $11^{\text {th }}$ EWTEC 2015, Nantes, France

[4] F. Biskup, P. Daus, R. Arlitt "Auslegung und Evaluierung eines Rotordesigns für Gezeitenströmungsanlagen“, $34^{\text {th }}$ Dresdner Wasserbaukolloquium, ISBN 978-3-86780-198-0, 2011

[5] P. Bösl, M. Arnold "Untersuchung des Added-Mass-Effekts unter turbulent instationären Bedingungen", thesis, University Stuttgart, Germany, Nov. 2014

[6] E. Bossanyi, P. Fleming, A. Wright "Validation of Individual Pitch Control by Field Tests on Two- and Three-Bladed Wind Turbines", IEEE Transactions on Control Systems Technology, Vol. 21, No 4, pp. 1067-1078, July 2013

[7] A. Broeren, M. Bragg "Spanwise Variation in the Unsteady Stalling Flowfields of Two-Dimensional Airfoil Models", AIAA Journal, Vol. 39, No. 9, pp. 1641-1651, September 2011

[8] T. Burton et.al. "Wind Energy Handbook", ISBN 978-0-471-48997-9, England, 2005

[9] T. Fischer "Mitigation of Aerodynamic and Hydrodynamic Induced Loads of Offshore Wind Turbines“, $\mathrm{PhD}$ thesis, University Stuttgart, ISBN 978-3-8440-1501-0, Germany, 2012

[10] R. Gasch, J. Twele et.al. "Windkraftanlagen: Grundlagen, Entwurf, Planung und Betrieb“, ISBN 978-3835101364, Germany, 2007

[11] Garrad Hassan "Tidal Bladed - Theory Manual", 2015

[12] R. Hoffmann "A comparison of control concepts for wind turbines in terms of energy capture", $\mathrm{PhD}$ thesis, Technische Universität Darmstadt, Germany, 2002

[13] J. Jonkman "Dynamic Modelling and Loads Analysis of an Offshore Floating Wind Turbine", PhD thesis, NREL/TP-500-41958, USA, Nov. 2007

[14] N. Kelley, B. Jonkman "TurbSim v1.06.00", NWTC Information Portal (TurbSim), https://nwtc.nrel.gov/TurbSim, Last modified 14October-2014, Accessed 29-January-2015

[15] A. Nieslony "Rainflow Counting Algorithm", Matlab Central Code Database, File ID \#3026, version 2010-04-03, 2003

[16] F. Menter, Y. Egorov "A Scale-Adaptive Simulation Model using Two-Equation Models", AIAA 2005-1095, 43 ${ }^{\text {rd }}$ AIAA Aerospace Sciences Meeting and Exhibit, Reno, USA, 2005

[17] P. Mutschler, B. Hagenkort, S. Joeckel "Control method for variable speed stall controlled wind turbine“, EWEC 1997, ISBN 09533392201 , pp. 542-545, Dublin, Ireland

[18] OpenHydro, Dublin, Ireland, 2014

[19] D. Schlipf "Lidar-Assisted Control Concepts for Wind Turbines", PhD thesis, University Stuttgart, Germany, 2015

[20] Schottel Hydro, Schottel Instream Turbine, Spay, Germany, 2014

[21] Voith Hydro Ocean Current Technologies, Voith HyTide ${ }^{\circledR} 1000-13$ Turbine, Heidenheim, Germany, 2014

[22] M. Wilkinson, K. Harman, B. Hendriks, F.Spinato, T. van Delft "Measuring Wind Turbine Reliability - Results of the Reliawind Project", EWEA 2011, Brussels, Belgium

[23] Z. Zhou, F. Scuiller, J.F. Charpentier, M.E.H. Benbouzid, T. Tang "Power Control of a Nonpitchable PMSG-Based Marine Current Turbine at Overrated Current Speed With Flux-Weakening Strategy", IEEE Journal of Oceanic Engineering, Issue 99, pp. 1-10, Oct. 2014 